\title{
Haptic Science Learning System for Students with Visual Impairments: A Preliminary Study
}

\author{
Takehiko Yamaguchi ${ }^{1}$, Steve Johnson ${ }^{1}$, Hyung Nam Kim ${ }^{2}$, Yueqing $\mathrm{Li}^{1}$, \\ Chang S. Nam ${ }^{1}$, and Tonya L. Smith-Jackson ${ }^{2}$ \\ ${ }^{1}$ Department of Industrial Engineering \\ University of Arkansas \\ 4207 Bell Engineering Center, Fayetteville, AR 72701, U.S.A. \\ \{tyamaguc, sajohns, yxl002, cnam\} @uark. edu \\ ${ }^{2}$ Grado Department of Industrial and Systems Engineering \\ Virginia Polytechnic Institute and State University \\ Blacksburg, Virginia 24061, USA \\ \{hykim7, smithjack\}@vt.edu
}

\begin{abstract}
This paper assesses the usability of developed haptic interface features to support future development of haptically enhanced science learning applications for students with visual impairments. Of the features developed and evaluated, three features are described in this study: Haptic Boundary, Menu Selection, and Object Recognition. Two focus groups participated in an interview and usability session. Results from this study give insight to the needs of the visually impaired community in regard to haptic applications.
\end{abstract}

Keywords: Haptic Virtual Environment, Science Learning, Universal Design, Assistive Technology.

\section{Introduction}

Special education programs support approximately 93,600 students with visual impairments, 10,800 of whom are students with both deafness and blindness [1]. The population of students with visual impairments increases when public schools are also factored in. In fact, most students with visual impairments (i.e., 92\%) are enrolled in public schools. Students with visual impairments should be given full access to the most appropriate technologies so that they can be given equal opportunities for learning in competitive educational settings [2].

Although great effort has been made to support inclusive educational environments for students with visual impairments, the effectiveness remains in doubt. A recent study found that $90 \%$ of science instructors primarily rely on a textbook-centered teaching style in their classes [3]. Even if certain assistive technology applications (e.g., Braille) are employed in a class, especially a science class, a challenge still exists among students with visual impairments in comprehending visually complex scientific concepts. In general, disciplines of science, technology, engineering, and mathematics (STEM) incorporate many visually complex concepts, such as diagrams 
and graphs $[3,4]$. These image constructs are not successfully represented by today's assistive technology applications [5, 6, 7].

A number of today's researchers put forth efforts to overcome the shortcomings of existing technology (e.g., Braille). They attempt to take full advantage of capabilities in the remaining modalities of those with visual impairments, such as a sense of touch (i.e., haptic). The results of their studies support that haptic technology contributes to the improvement of understanding graphic-based information [8, 9, 10]. Haptic applications have the potential to be useful for students with visual impairments to learn visually complex scientific concepts. However, very little research has been conducted on the development of user interface design guidelines or user friendly features for haptic systems. Although a number of graphical user interface design guidelines are available (e.g., W3C, Section 508), their compatibility to haptic systems is questionable.

This paper aimed to contribute to the enhancement of haptic user interfaces particularly of a science education learning application. More precisely, haptic interface features were developed to allow students who are visually impaired to effectively interact with a haptic-embedded educational application by using their auditory and haptic sensory modalities. These haptic features were formatively evaluated for usability and accessibility through focus groups.

\section{Main Haptic Interface Features}

Development of a haptic science learning application began with observational field work that aided the development team in assessing the unique needs of students with visual impairments. The field study would then support development of a haptic feature set that ultimately would serve as the backbone for a future application. Though many versatile feature sets were developed, this paper focuses on three primary features: menu selection, object recognition, and haptic boundary.

\subsection{Requirements Analysis}

An initial field study was conducted to observe students with visual impairments in a science learning environment. The development team observed five high school students (grades 7-9) - all of whom had varying degrees of visual impairment - during an instructor-led frog dissection lab at a nearby state-funded school for the blind. From the observational field study, the development team was able to discern several key paradigms that would serve as the initial development for a feature set. These paradigms were that students with visual impairments need (1) vocal instruction, encouragement, and guidance, (2) physical guidance when necessary, (3) the ability to adjust visual aspects of the system to meet each student's level of visual impairment, (4) the ability to ask questions or receive aid in a task, and (5) to provide a sense of independence to overcome an individual's impairments.

The development team then created a scenario based-design based on the observation of interactions between students and teachers. This scenario based design would serve as an initial development model to assess the needs of all feature set tools. 


\subsection{Main Design Features}

Haptic Boundary. To provide students with visual impairments with a navigational tool, a Haptic Boundary feature was developed to "sandbox" the interactional space for a user within the virtual environment. The Haptic Boundary can also be utilized to gain spatial awareness within the virtual environment, as all haptic interactions take place inside of this rectangular constraint. Figure 1 diagrams the Menu Selection feature (further discussed below) with its invisible Haptic Boundary component around the virtual environment.

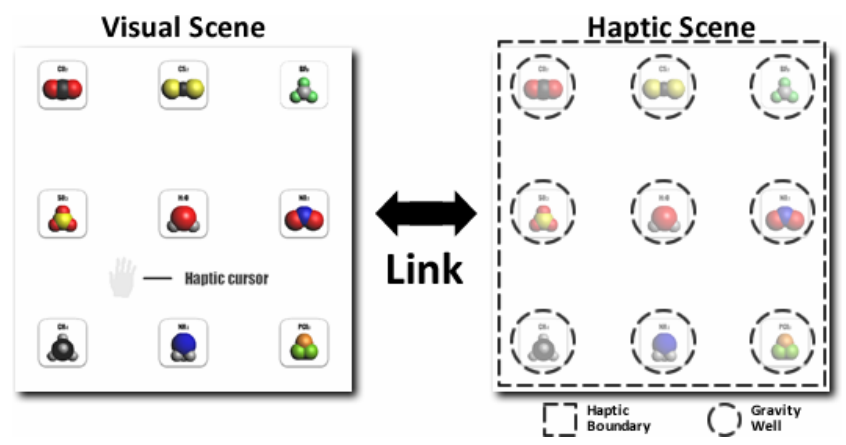

Fig. 1. Matrix type interface for menu selection with haptic boundary and gravity well

Menu Selection. To provide students with visual impairments with a means of object selection, a Menu Selection feature was developed with an emphasis on ease of use in regard to navigation, content organization, and selection. Though multiple interface types were designed for future development, the focus of this study was Type 1: Matrix Menu Selection (see Figure 1). In this interface type, selectable buttons are arranged in a grid-like pattern, similar to that of an Excel Spreadsheet. Each button represents a particular molecule and contains a haptic gravity well component. In haptic terms, a gravity well is an invisible haptic object that reacts when a user's cursor is near by pulling and snapping the user's cursor to its center both visually and haptically. Around the entire menu is a haptic boundary component. Figure 1 diagrams Interface Type 1: Matrix Selection with 9 selectable buttons - each corresponding to a particular molecule - as well as its invisible haptic components. The Menu Selection feature contains the following components:

\section{(a) Visual Components}

- 3D Hand Cursor - This is the cursor operated by the connected haptic device. The cursor appears as a 3D hand that animates in when feeling/interacting with objects.

- Selectable Buttons - These are arranged in a 3x3 grid on the page. Each button displays a custom graphic and a textual description that correspond to a particular molecule.

\section{(b) Sound Components}

- Navigational Audio: Synthetic Voice instructing the user on how to use the Menu Selection feature. 
- Button Hover audio : This audio plays each time a user hovers over a particular button in order to alert the user as to what button a user hovers over (e.g. Hovering over the $\mathrm{CO}_{2}$ button results in audio feedback " $\mathrm{CO}_{2}$ : Carbon Dioxide")

- Button Selection Audio: Synthetic Voice (i.e. "You have selected $\mathrm{CO}_{2}$ : Carbon Dioxide")

\section{(c) Haptic Components}

- Gravity Well - Each selectable button in the Main Menu contains a Gravity Well. When the user's cursor is within a Gravity Well pull radius, the user's cursor will visually and haptically snap with an attractive force to the center of the particular molecule button.

- Haptic Boundary - The virtual environment contains a haptic bounding box that defines the edges of the virtual environment and can be used by the user for navigational purposes. If the cursor touches the haptic boundary, a spring force is presented that prohibits user movement outside of application screen. Also, a small vibrotactile feedback is produced when the user's cursor comes into contact with the wall.

Object Recognition. To provide students with visual impairments with a means of interacting and recognizing haptically rendered objects, an Object Recognition feature was developed to render simple and complex molecular objects in both 2-Dimensions (2D) and 3-Dimensions (3D). Figure 2 diagrams each of the molecular models that a user can interact with for the molecule $\mathrm{H}_{2} \mathrm{O}$ : Water.

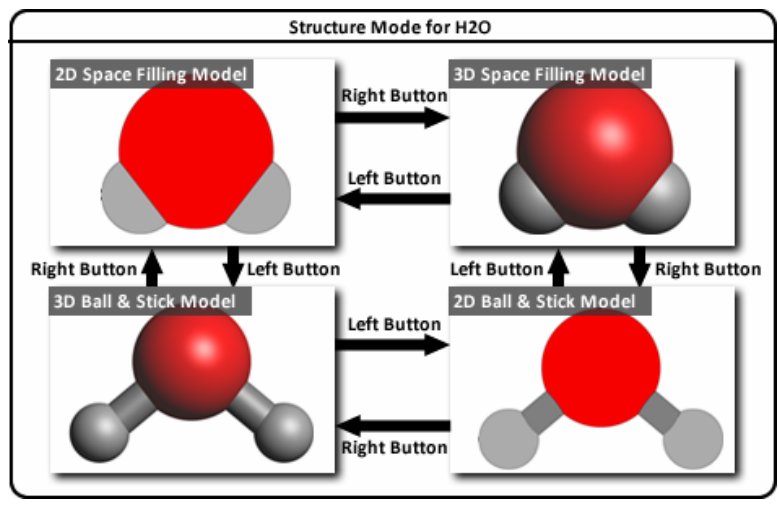

Fig. 2. Haptic models supported by the molecule $\mathrm{H}_{2} \mathrm{O}$ : Water

Fourteen molecules were planned for development. However, at the time of this study, only two molecules were supported: $\mathrm{H}_{2} \mathrm{O}$ : Water and $\mathrm{CO}_{2}$ : Carbon Dioxide. For each supported molecule, two molecular model types (i.e. Space Filling Model and Ball \& Stick Model) were developed and rendered in both 2D and 3D. Through the use of a haptic device, users are able to feel each molecular model in a virtual environment as if it were a real physical object. The Object Recognition feature contains the following components: 


\section{(a) Visual Component}

- 3D Hand Cursor - This is the cursor operated by the connected haptic device. The cursor appears as a 3D hand that animates in when feeling/interacting with objects.

- Molecular model - This is one of four models - 2D Space filling model, 3D Space filling model, 2D ball \& stick model, or 3D ball \& stick model.

(b) Sound Component

- Instructional \& Navigational Audio - Synthetic Voice describing which model the user was interacting with (i.e. "2D Ball and Stick Model”).

\section{(c) Haptic Component}

- Haptic Boundary - The virtual environment contains a haptic bounding box that defines the edges of the virtual environment and can be used by the user for navigational purposes. If the cursor touches the haptic boundary, a spring force is presented that prohibits user movement outside of application screen.

- Haptic Molecular Model - Depending on the selected molecule, a haptic Component of the molecular model is presented in the virtual environment for the user to haptically interact with using their haptic device.

\section{Method}

Two focus groups - one for teachers (Focus Group 1) and another for students (Focus Group 2) - participated in a hands-on demonstration of haptic equipment and design features followed by a question/answer session regarding their suggestions, opinions, and experiences in order to assess the usability of supported haptic devices as well as newly developed design features. To reiterate, though many versatile feature sets were developed, this paper focuses on three primary features: menu selection, object recognition, and haptic boundary.

\subsection{Participants}

Focus Group 1 consisted of 2 male and 2 female teachers. All but one of the teachers were sighted, with one female teacher experiencing severe visual impairment. Teachers were between 31 and 35 years of age. Focus Group 2 consisted of 3 male and 1 female students. All but one of the students were completely blind, with one male student having partial vision in one eye. Students were between 14 and 17 years of age.

\subsection{Apparatus}

The developing setup used a 3.4 GHz Pentium R, 1.0GB of RAM PC, with an Adobe Flash CS3 for the visual rendering and a $\mathrm{C} / \mathrm{C}++$ Novint SDK for the haptic rendering. Two haptic devices were used in the present study: Phantom Omni and Novint Falcon. The haptic features were developed in a multithreaded way, in which graphics rendering was of $60 \mathrm{~Hz}$ and haptic control was updated at a rate of $1 \mathrm{kHz}$. 


\subsection{Procedure}

Focus Group 1 first participated in a hands-on demonstration of haptic devices via the use of accompanying device demos. Second, a hands-on demonstration of the developed haptic features was conducted. Finally, a question/answer session was conducted with the following questions: (1) What are the most difficult science concepts for your students?, (2) What ideas could you provide regarding the usefulness of haptic products such as the Phantom Omni and the Novint Falcon in the context of science learning?, (3) In what ways could our developed haptic features be enhanced or improved in order to support science learning?, and (4) What ideas could you provide about further enhancements or features that could be developed in order to increase the usability of a future developed science learning application by students with visual impairments?

Focus Group 2 first participated in a hands-on demonstration of haptic devices via the use of accompanying device demos. Second, a hands-on demonstration of the developed haptic features was conducted. Finally, a question/answer session was conducted with the following questions: (1) What are the most difficult science concepts for you to learn?, (2) What ideas could you provide regarding the usefulness of haptic products such as the Phantom Omni and the Novint Falcon in the context of science learning?, (3) In what ways could our developed haptic features be enhanced or improved in order to support science learning?, and (4) What ideas could you provide about further enhancements or features that could be developed in order to increase the usability of a future developed science learning application?

\section{Results}

This section summarizes comments and suggestions that obtained from the Teacher and Student Interviews regarding the developed haptic features (see Table 1).

\subsection{Menu Selection}

Comment 1: Main Menu buttons should encompass the whole screen and, as a whole, fill the entire haptic area so that there is no "Extra" surrounding space.

Proposed Design Change: Increasing the size of the Main Menu navigation buttons would make it easier to navigate the Main Menu instead of wasting time having to locate selectable buttons within the surrounding menu space.

Comment 2: The integration of Hot Keys or commands could improve usability for users that are visually impaired students, as most users with visual impairments are adept at utilizing the keyboard on a day to day basis.

Proposed Design Change: Keyboard Hot Keys or Function activated features should be integrated. For example, "H20" could be typed rather than clicking the H20 Button.

\subsection{Object Recognition}

Comment 1: Vibratory/tactile differences should be presented interacting for 2D and 3D haptic models Vibratory/Tactile differences help users with visual impairments to more easily distinguish haptic elements within the virtual environment. 
Proposed Design Change: Provide unique tactile or vibratory feedback for each element within the virtual environment.

\subsection{Haptic Boundary}

Comment 1: Unique vibratory feedback should be presented in order to distinguish the haptic boundary from other haptic elements within the virtual environment.

Proposed Design Change: To separate the Haptic Boundary from other haptic objects within the environment, the Haptic Boundary needs to produce a unique vibratory feedback when the user comes into contact with the boundary.

Comment 2: Haptic Boundary needs improvement As the Haptic Boundary was only present for certain positions on the $\mathrm{Z}$-axis in the $3 \mathrm{D}$ virtual environment; diminishing each of use and creating uncertainty for end users.

Proposed Design Change: The Haptic Boundary will be redesigned in order to maintain haptic consistency around the $3 \mathrm{D}$ virtual environment.

Table 1. A summary of users' comments. (Note. MMS: Main Menu Selection, OR: Object Recognition, HB: Haptic Boundary, T: Teachers, and S: Students).

\begin{tabular}{|c|c|c|c|}
\hline Design Feature & User & Description & $\begin{array}{c}\text { Number of } \\
\text { comment }\end{array}$ \\
\hline \multirow[t]{2}{*}{ MMS } & \multirow[t]{6}{*}{$\mathrm{T}$} & $\begin{array}{l}\text { Main Menu buttons should encompass the whole } \\
\text { screen and, as a whole, fill the entire haptic area so } \\
\text { that there is no "Extra" surrounding space. }\end{array}$ & (1) \\
\hline & & $\begin{array}{l}\text { The integration of Hot Keys or commands could } \\
\text { improve usability for users that are visually impaired } \\
\text { students, as most of them are adept at utilizing the } \\
\text { keyboard }\end{array}$ & (2) \\
\hline \multirow[t]{3}{*}{ OR } & & Device cursor should be the larger. & $(3)$ \\
\hline & & $\begin{array}{l}\text { A User wants to change the amount of force using } \\
\text { zooming function. }\end{array}$ & (4) \\
\hline & & $\begin{array}{l}\text { It's difficult to recognize size of atom and relationship } \\
\text { between each atom. }\end{array}$ & (5) \\
\hline $\mathrm{HB}$ & & $\begin{array}{l}\text { Haptic Boundary needs improvement as it was only } \\
\text { present for certain positions on the Z-axis in the 3D } \\
\text { VE; diminishing each of use and creating uncertainty } \\
\text { for end users. }\end{array}$ & (6) \\
\hline MMS,OR,HB & $\mathrm{S}$ & Sound effect should be played. & (7) \\
\hline MMS,OR,HB & \multirow[t]{3}{*}{$\mathrm{T} / \mathrm{S}$} & Instruction should be played quickly. & (8) \\
\hline OR,HB & & $\begin{array}{l}\text { Vibratory/tactile differences should be presented } \\
\text { interacting for } 2 \mathrm{D} \text { and } 3 \mathrm{D} \text { haptic models. Such } \\
\text { differences help users with visual impairments to } \\
\text { more easily distinguish objects within the virtual } \\
\text { environment. }\end{array}$ & (9) \\
\hline OR & & $\begin{array}{l}\text { It's difficult to recognize the shape of 3D Ball \& Stick } \\
\text { model. }\end{array}$ & (10) \\
\hline
\end{tabular}




\section{Discussion}

The development team will enhance the developed haptic features based on the comments received from the Focus Group Interviews. In this section, user comments have been categorized in order to extract a use-case scenario for design changes.

\subsection{Visual Representation}

\section{a. Menu Button Size}

- The size of the navigation buttons within the Menu Selection Feature should be increased so that the total space occupied by the navigational buttons fills the entire screen. This would allow for easier Menu Selection navigation and reduce the amount of wasted time the user needed to locate selectable buttons within the surrounding menu space (based on T/S's comment (1)).

\section{b. Object Size}

- A larger cursor surface area would allow for easier haptic interaction. (based on T/S's comment (3)).

- Magnification functionality could help to cater to students with a variety of visual impairments. For example, some kids need large object, other need extremely small objects. Allowing the user to zoom in and out could be very helpful(based on T/S's comment (4),(5)).

\subsection{Audio Representation}

\section{a. Audio Duration}

- Sound instruction should be played as quickly as possible(based on T/S's comment (8)).

\section{b. Help Function}

- A user initiated Help Function (press ' $\mathrm{H}$ ' for instructional audio) feature should be developed to allow beginner users to listen to instructional or supplemental audio in order reduce unnecessary audio instruction for advanced users. (based on T/S's comment (8)).

\section{c. Sound Effect}

- Audio feedback - either differing pitches or subtle sound effects - should be incorporated in order to enhance system usability. (based on T/S's comment (7)).

\subsection{Haptic Representation}

\section{a. Tactile Effect}

- When a user touches an object in the environment, a difference in tactile or vibratory feedback would help users haptically distinguish objects within the environment. (based on T/S's comment (9)). 


\section{b. Cursor Guidance}

- The Haptic Boundary will be redesigned in order to maintain haptic consistency around 3D virtual environments. (based on T/S's comment (6)).

- Objects that have cursor guidance capabilities (i.e. a magnetic object that causes the user's cursor to "stick" to the object) can improve system usability. (based on T/S's comment (10)).

\subsection{Other Functions}

\section{a. Keyboard Functionality}

- Keyboard Hot Keys or commands should be integrated. For example, "Esc" would be equivalent to our current "Return" button. Or being able to type "H 2 0" or " 1 " and entering Structure Mode for the H20 molecule.

And then, we tried to change design of main design feature based on the categorized results. In Main Menu Selection we should revise Visual representation; Screen Space, Audio representation: Duration time, Repeatability, and Sound Effect, and Haptic representation: Tactile Effect. In Haptic Boundary design feature, we should revise Audio representation: Sound Effect, and Haptic representation: Tactile Effect, and Force-feed Back Effect. In Object Recognition main design feature, we should revise Visual representation: Object size, Audio representation: Duration time, Repeatability, and Sound Effect, and Haptic representation: Tactile Effect, and Forcefeed Back Effect.

\section{Conclusion}

The main goal of the present study was to assess the usability of a set of developed haptic features as well as ascertain other improvements and ideas for undeveloped design features. To assess the usability of developed haptic features, two focus group usability and interview sessions were conducted with teachers and students from a local state-funded school for the blind.

The suggestions and comments obtained from the focus group interview and usability sessions were categorized and classified in order to extract use case scenarios for future developed features. Comments and suggestions will also be used to reassess future application based design goals. Future work will include refining haptic effects, incorporating new modalities, and assimilating new haptic features into a beta version of the system.

Acknowledgement. The authors thank teachers, students, and administrators at the Arkansas School for the Blind, Little Rock, AR, for their collaboration. This project was supported by National Science Foundation grant DRL-0736221 to Chang S. Nam (University of Arkansas) and Tonya L. Smith-Jackson (Virginia Tech). Any opinions, findings, conclusions, or recommendations expressed herein are those of the authors and do not necessarily reflect the views of the National Science Foundation. 


\section{References}

1. American Foundation for the Blind. Statistics and sources for professionals, http: / / www . afb . org/Section. asp? SectionID=15\&Document ID=1367

2. Riemer-Reiss, M.: Assistive technology use and abandonment among college students with disabilities. International Electronic Journal for Leadership in Learning 3(23) (1999)

3. Kumar, D., Rangasamy, R.: Science for students with visual impairments: Teaching suggestions and policy implications for secondary educators. Journal of Science Education 5(3) (2001)

4. Fitzpatrick, D.: Teaching science subjects to blind students. In: Seventh IEEE International Conference on Advanced Learning Technologies, pp. 917-918 (2007)

5. Aldrich, F.K., Parkin, A.J.: Tape recorded textbooks for the blind: A survey of producers and users. The British Journal of Visual Impairment 6(1), 3-6 (1998)

6. Karshmer, A., Gillan, D.: How well can we read equations to blind mathematics students: Some answers from psychology. In: Stephanidis, C. (ed.) Proceedings of the 2003 Human Computer Interface International Conference, pp. 1290-1294. Lawrence Erlbaum, New Jersey (2003)

7. Yu, W., Reid, D., Brewster, S.: Web-based multimodal graphs for visually impaired people. In: Proceedings of the 1st Cambridge Workshop on Universal Access and Assistive Technology, pp. 97-108. Springer, Cambridge (2002)

8. Brewster, S.: Visualization tools for blind people using multiple modalities. Disability and Rehabilitation 24(11), 613-621 (2002)

9. Liffick, B.W.: A haptics experiment in assistive technology for undergraduate HCI students. In: Proceedings of the HCI International 2003 conference, pp. 1300-1304 (2003)

10. Sjostrom, C., Danielsson, H., Magnusson, C., Rassmus-Grohn, K.: Phantom-based haptic line graphics for blind persons. Visual Impairment Research 5(1), 13-32 (2003) 